\title{
Kepler's Tertius Interveniens
}

\section{Ken Negus}

Tertius Interveniens, written in 1610, is one of Kepler's most powerful and passionate treatises on astrology, written as a defence of the subject against extremists on both sides, on the one hand those who would condemn astrology altogether, and on the other those who accepted everything said and done in its name, no matter how preposterous. Hence he is the 'third party intervening', as indicated by the title.

The following extract is near the centre of the book, and is otherwise 'central' as an expression of the main tenets of Kepler's thought on astrology. In this short passage, he comments incisively on the following topics: the non-material, 'spiritual' nature of astrology; geometry as the all-embracing archetype through which the messages of the sky are communicated to earth; the horoscope as indelible 'imprint' on the soul of the infant being born; astrology as 'music'; the rationale of progressions and directions (symbolic measures used in prediction); astrological genetics; transits and mundane (political and historical) astrology.

Not to be forgotten when reading Kepler's arguments concerning astrology is his major role in the history of science. Although he is remembered primarily as an astronomer he also had much to say about biology, geology, meteorology, medicine and many other areas. His philosophical thinking also suggests much that is far ahead of his time, including prefigurations of Jungian psychology (archetypes and the collective unconscious). But let him, in slightly abridged form, speak for himself: ${ }^{1}$

Thesis 64. All powers coming down from above are ruled according to Aristotle's teaching: namely, that inside this lower world or earthly sphere there is a spiritual nature, capable of expression through geometry. This nature is enlivened by geometrical and harmonic connections with the celestial lights, out of an inner drive of the Creator, not guided by reason, and itself is stimulated and driven for the use of their powers. Whether all plants and animals, as well as the Earth's sphere, possess this faculty, I

Negus, Ken, 'Kepler's Tertius Interveniens, Culture and Cosmos, Spring/Summer 1997, Vol. 1 no 1, pp. 51-4. 
cannot say. It is not an unbelievable thing, for they have various faculties of this kind: in that the form in every plant knows how to put forth its adornment, gives the flower its colour, not materially, but formally, and also has a certain number of petals; nor [is it unbelievable] that the womb, and the seed that falls into it, has such a marvellous power to prepare all the body parts in appropriate form....The human being, however, with his soul and its lower powers has such an affinity with the heavens, as does the surface of the Earth, and this has been tested and proven in many ways, of which each is a noble pearl of astrology, and is not to be rejected along with [all of] astrology, but to be diligently preserved and interpreted.

Thesis 65. Above all, I might in truth flatter myself with having experienced this observation: that the human being, in the first igniting of his life, when he now lives independently for the first time, and can no longer live in the womb, receives the character and formation of the sky's whole stellar configuration, or the form of the conflux of radii on earth, and maintains it unto his grave. Afterwards this can be perceived in the formation of the face and the remaining bodily structure, as well as in the person's behaviour, habits and gestures, so that he might create, with his bodily form, corresponding attraction and charm for himself in the eyes of other people, and with his actions bring forth corresponding fortune. Then thereby (as well as from the mother's fantasies before the birth and from the rearing of the child thereafter), a great difference from other people is created, so that one person is brave, cheerful, joyful, selfconfident; and another lethargic, lazy, neglectful, shy, forgetful, hesitant, and whatever other general characteristics there may be, which can be compared with configurations that are pleasant and exact, or complex and awkward, or also with the colours and motions of the planets. This character is not received into the body - for it is much too ungainly for that - but into the nature of the soul itself, which behaves like a point, so that she [the soul] might be transformed in points of the conflux of radii; and not only do these points impart reason to her, from which we human beings might be called reasonable above all other creatures, but she is to grasp in the first moment another kind of implanted reason - geometry in the radii as well as in musical sounds [i.e. 'voices,' in the technical musical sense], without a lengthy learning process.

Thesis 66. Second, so it is with every plant, that it is on schedule when it is to ripen or blossom. This time is prescribed to it at its creation, and by 
external warmth and other means it is lengthened or shortened, but can never be totally altered. In like manner the human being's nature, upon entry into life, receives not only an instantaneous image of the sky, but also its movement, as it appears down here on earth for several days in a row, and in certain years derives from this moment the manner of outpouring this or that humour; these years are precisely and sharply indicated, based on the projection of the first few days [of life]. This is a truly marvellous thing, and is like an image or outflowing of the natural proportion of a day to a year. Thus this short time or 'tempus typicum' in human nature with all its parts is multiplied by 365 ; and all of natural life, out of this multiplication, remaining rigidly in its memory, is deducted and unwound as from a ball of yarn, so that then the whole future life, insofar as it deals with natural matters, in the course of a quarter-year is wound up and stored in a little bundle. Such a causality and natural proportion cannot, however, be applied to the profections, ${ }^{2}$ for not the Ascendant and not the Sun, but Jupiter makes its revolution in 12 years, as the Moon does in 28 days, and accordingly the best of the profections should be assigned to the transits; the rest is useless noise. I have often harboured the thoughts that there is nothing to directions because we must reach out so far for their causality, and one cannot accommodate them any differently. But I must confess that nonetheless the causality resembles nature, because it requires a natural proportion; and that our experience is so clear, that they are not to be denied as true for the astrologers.

Thesis 67. Third: this is a curious thing that the nature [of the human being], which receives this character [of the sky], also favours its relatives by some similarities in the celestial constellations. When the mother is great with child and her time has come, then nature seeks out a day and hour for the birth that is comparable celestially with that of the mother's father or her brother.

Thesis 68. Fourth, every [human] nature knows not only its celestial character, but also every day's configurations and motions in the sky so well, that as often as a transiting planet comes into its character's Ascendant or other prominent place, especially into radical points, it [the nature] accepts it and is thereby variously affected and stimulated.

Thesis 69. Fifth, there is also the experience that every strong conjunction, by itself, without considering the relationship to a particular 
54

Kepler's Tertius Interveniens

person, stimulates people in general (where a nation lives together in an ordered society), and makes them capable of acting as a community that is unified just as the stars then shine together. This was discussed in detail in my book De stella serpentarii. Thus I have seen many examples of epidemics in which the humours are stirred up more when strong stellar configurations are present (that is to say, the human natures are stimulated to drive out the humours). In like manner all these points - and many more could be cited, being from the same cause - and the possibility of one coming from the other could be proven and defended.

\section{References}

1. Translation by Ken Negus, 1997.

2. A forecasting technique in which the houses of the horoscope represent the years of life, beginning with the first house and the first year of life. 